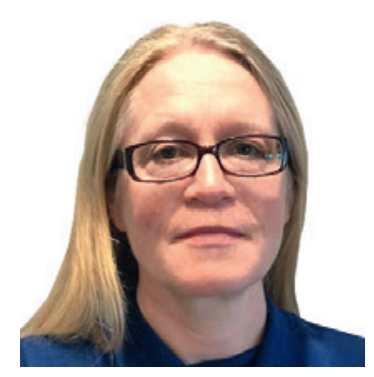

\title{
Health Apps in Primary Care
}

\author{
Trude M. Backer Mortensen
}

Nordre Follo rådhus, Ski, Norway

Abstract from Wangler J, Jansky M: The use of health apps in primary care - results from a survey amongst general practitioners in Germany. Wien Med Wochenschr 2021;171:148-156.

\section{Keywords}

Health apps · Health promotion · Prevention · Primary

healthcare $\cdot \mathrm{mHealth}$

\begin{abstract}
Mass availability and use of health apps raises the question as to how they might be integrated into healthcare systems towards improving prevention and therapy. This study has researched prevailing opinion on health apps amongst primary care physicians, potential application areas physicians have seen in their experience with these apps up to now, and situations suitable for using apps in patient care. A total of 2138 primary care physicians in the state of Baden-Württemberg, Germany, responded to an anonymised written survey between March and June 2020. Physicians with a positive opinion (36\%) emphasised motivation and compliance as advantages, whereas sceptical respondents (43\%) expressed suspicion regarding data privacy and reliability as well as
\end{abstract}

legal issues and additional workload arising from using the apps. Even so, a clear majority accepted the potential benefit from sensible use of health apps with features providing prevention and lifestyle support (90/76\%). With respect to patients using the apps, $54 \%$ of respondents saw a positive contribution to healthcare and/or recovery. Despite the perceived benefits of health apps, general practitioners are still reluctant to bring up or recommend health apps in their consultations. Many physicians do not feel capable of giving expert advice to patients on the apps available. Many general practitioners are aware of the potential that health apps may have in improving prevention and treatment. However, there are reservations and uncertainties regarding clarity, transparency, and privacy issues in these apps. More focus should be placed on these concerns to ensure ideal conditions for integrating health apps into primary care.

(c) 2021 The Author(s) 


\section{Knowledge Transfer}

\section{Apps in Primary Care}

There are a number of different apps related to health and lifestyle. These are offered by different players, and their quality frequently depends on who created the content. Some of the apps provide pure information about a topic, others require that you enter personal and health-related data, while still others process quantitative data from the user. Thus, there are several aspects to consider when using/recommending apps. For example, it is important that the information provided by an app is correct and adapted, that personal data is processed according to current rules, and that quantitative data is of good enough quality/measurement tools are used correctly and provide measurements suitable for further use. In the jungle of apps, individual GPs or other health professionals cannot be expected to keep up to date with which apps are good and how they work.

\section{Lifestyle Apps}

When it comes to nutrition, there are many apps that calculate the energy level in food for you. This in itself is useful as the alternative requires a lot of time and knowledge. These apps rarely include micronutrient or protein content and thus will not provide an overall assessment of the diet. Furthermore, data regarding nutrient content of different foods may vary depending on where they are grown/produced [1-3], and information on this will not necessarily be made available. Another challenge is portion size. Often the amount must be entered as weight which makes correct use difficult. Alternatively, the app may be based on portions but their size may be inconsistent with what the user actually eats and drinks [4]. Uncertainty about whether quantity of food should be stated as dry/raw or cooked weight (e.g., pasta or rice) can also have an impact on the end result.

In addition to these challenges, apps may give the user a moment of realisation by showing in black and white what it means to choose whole fat toppings versus lean, what a bag of potato chips provides in energy compared to a meal etc.; however, what you do not see is that a topping of sardines provides a healthier type of fat than a full fat cheese, that nuts and olives provide healthier fats in spite of their high energy content as well as fibre and micronutrients compared to e.g. potato chips, and that dried fruit provides a lot of energy and sugar but also fibre and micronutrients compared to e.g. sweet treats.

Similar duality can be found where physical activity is included in the app with uncertainty around calculations and opportunities for learning.

\section{Intolerance Apps}

Apps that provide information on ingredients contained in food that can be the cause of food intolerance are an example of tools where the country of origin can be important for nutritional content. Varying species used in different parts of the world, cultivation conditions, seasonal variations, and production methods can some- times have an impact on the final content of certain components in the food and/or the reaction of the individual. Provided that the app uses food data that corresponds to where the user lives, it can be a useful aid. Some of these apps have "scanner functions" that make spotting food easier.

Also, apps that provide the content of food components require guidance from health professionals. In the case of irritable bowel syndrome, for example, an app that informs about the amount of FODMAPs in food will be useful initially/during the period of elimination, but if the app does not differentiate between the different FODMAPs it will be less beneficial as food is reintroduced. Also, these apps are of little help with regards to an overall healthy composite diet.

\section{Malnutrition Apps}

There are examples of apps that help calculate the intake of energy and protein with a view to preventing malnutrition. In Norway, a group affiliated with the University of Oslo, created such an app that also has a web-based decision support function for health professionals. The app has so far only been tested within the specialist health service [5]. Testing in the primary health service is planned for autumn 2021.

\section{Summary}

If GPs and other health professionals are to use/recommend apps, several aspects must be considered. Examples include a public approval scheme related to privacy rules, quality certification according to defined points, and possible tariffs that healthcare professionals can use when apps are included in the treatment [6]. In addition, the possibility of interaction between app/web-based tools and electronic patient records is of great importance. If data from app/web-based tools must be manually transferred to a medical record, extra work is created which in turn reduces the chance of use in health care.

For nutrition apps without a decision support system, qualified follow-up by a dietitian or other competent healthcare professionals will still be necessary. The apps provide information and contribute to the collection of data. However, they do not replace the assessment of total intake based on individual circumstances and needs nor are they able to propose appropriate measures. Apps with a decision support system are being tested and may in the long run be a better tool.

\section{Disclosure Statement}

I hereby declare that there are no conflicts of interest with regard to this commentary. 


\section{References}

1 Merchant AT, Dehghan M: Food composition database development for between country comparisons. Nutr J. 2006;5:2.

2 Lupiañez-Barbero A, González Blanco C, de Leiva Hidalgo A: Spanish food composition tables and databases: need for a gold standard for healthcare professionals. Tablas y bases de datos de composición de alimentos españolas: necesidad de un referente para los profesionales de la salud. Endocrinol Diabetes Nutr (Engl Ed). 2018;65(6):361-373.

3 Champagne CM, Wroten KC: From food databases to dietary assessment: A beginning to an end approach for quality nutrition data. Nutrition \& Dietetics. 2021;69:187-194.

4 Tay W, Kaur B, Quek R, et al.: Current Developments in Digital Quantitative Volume Estimation for the Optimisation of Dietary Assessment. Nutrients. 2020;12(4):1167.
5 Paulsen MM, Varsi C, Andersen LF: Process evaluation of the implementation of a decision support system to prevent and treat disease-related malnutrition in a hospital setting. BMC Health Serv Res. 2021;21:281.

6 Wangler J, Jansky M: The use of health apps in primary care - results from a survey amongst general practitioners in Germany. Wien Med Wochenschr. 2021:171:148-156.

Correspondence to:

Trude Backer Mortensen, trude.backer.mortensen@nordrefollo.kommune.no 